\title{
Development and Usability Testing of 'HELP': An Information Intervention for Parents of Children with Newly Diagnosed Acute Lymphoblastic Leukaemia
}

\author{
Bryan, G1. Vindrola, C22. Harding, V3. Ranasinghe, N4. Richardson, A55. Samarasinghe, N6. Taylor, R7 . Gibson, F6,8.
}

Introduction:

Parents of children newly diagnosed with Acute Lymphoblastic Leukaemia (ALL) experience a high care burden. Treatment for ALL is mainly outpatient based and parents require training in administrating medicines, handling central lines, and symptom management (Flury et al, 2011). In addition, parents have to be consented to extremely complex treatment protocols (Eder et al, 2007). In our previous research parents described receiving minimal support to facilitate the acquisition of new knowledge about their child's care and treatment (Gibson et al, 2018).

\section{Objectives:}

To develop and usability test an information intervention for parents of children with ALL.

Methods:

3. Feasibility Testing

HELP underwent feasibility testing with a

diverse group of HCPs and parent

representatives. Feedback was provided via

interview $(\mathrm{N}=5)$ or via written text $(\mathrm{N}=2)$

\section{Refinement Phase 1}

HELP refined using feedback. HELP V2 created. Videos were filmed with parents of children who had finished treatment $(\mathrm{N}=2)$ and a Haematology consultant.

\section{Development Phase}

HELP was developed using a stepwise approach in collaboration with Health professionals (HCPs) and parent representatives. HELP incorporates text, links to resources and videos. Multiple meetings with key stakeholders. Cheese and wine evenings and breakfast meetings held to discuss content and encourage staff to contribute to website text.

\section{Previous research}

Parents of children with ALL told us that they received minimal support to facilitate their acquisition of knowledge about their child's disease and treatment (Gibson 2018).

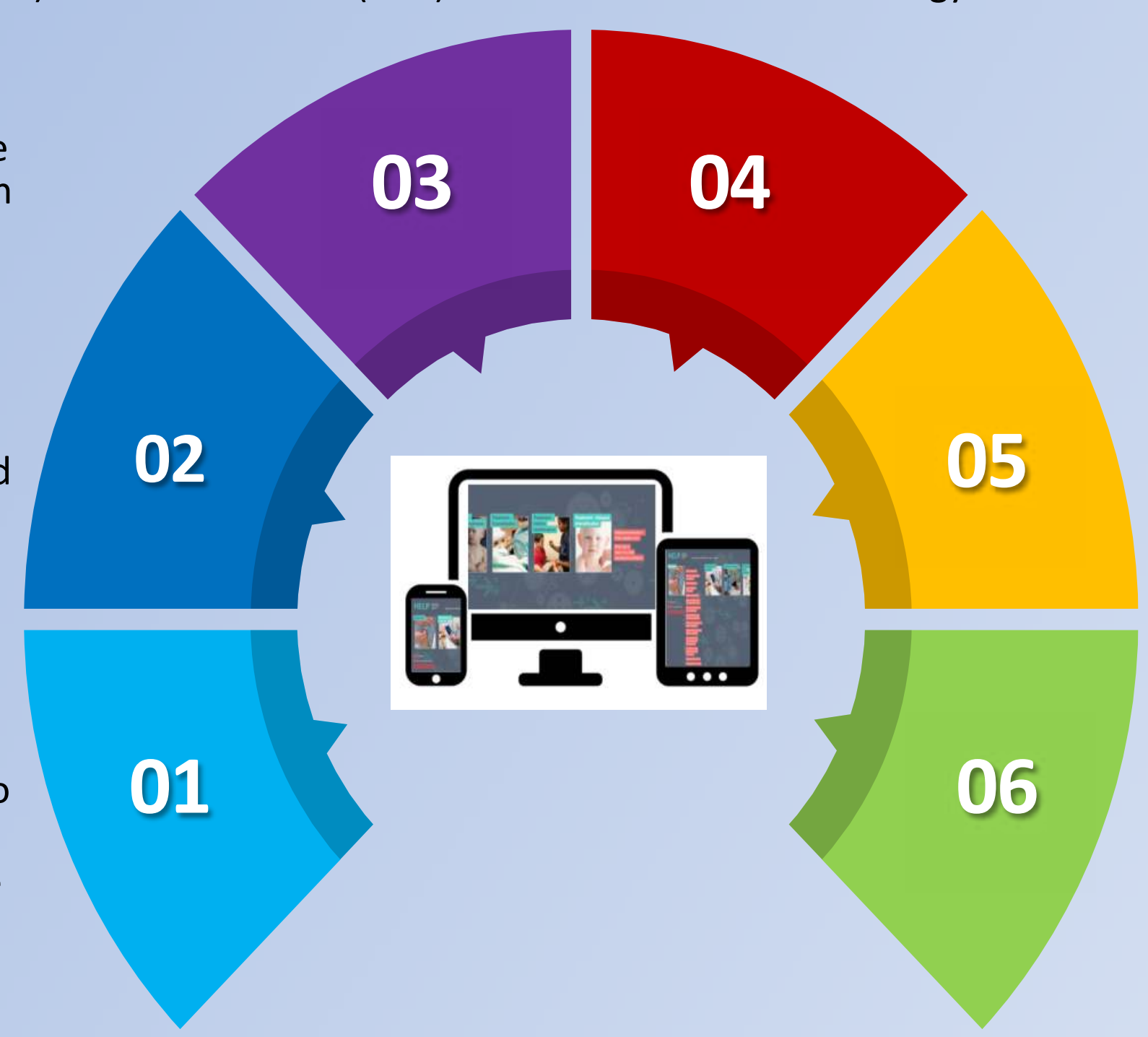

HELP underwent usability testing with Parents of children $>6$ months post diagnosis $(N=7,3 M, 4 F)$. Participants were White British and were native English speakers. Parents used HELP on a IPad while Talking Aloud, completed the System Usability Scale (SUS) and took part in a semi-structured interview.

\section{Refinement Phase 2}

HELP refined using feedback. HELP V3 created.

\section{Results:}

Fig. 1: The Six Phases in the Development of HELP

Initial development of HELP was slow. Engaging and recruiting HCPs to the development and feasibility testing phases of this study was challenging. Filming videos with parents and HCPs, proved worthwhile as parents responded favourably to videos during usability testing. Data reports on user performance and parent satisfaction were collected. Parents reported that they found HELP easy to use and easy to learn how to use. Parents were positive about HELP content, but some parents disliked the layout of information and the colours used on the resource. Overall, parents stated that they would have found the resource useful.

\section{Conclusion:}

The HELP website has been developed after extensive engagement with parents and input from HCPs. It has now undergone feasibility and usability testing. We now have a resource that we are confident will meet the information needs of parents of children with ALL.

\section{References}

Eder et al, 2007. Improving informed consent: suggestions from parents of children with leukemia. Pediatrics, 119 (4), e849-e859; Flury et al. 2011. Experiences of Parents with Caring for their Child after a Cancer Diagnosis. Journal of Pediatric Oncology Nursing, 28 (3), 143-153; Gibson et al, 2018. Insights from parents of a child with leukaemia and healthcare professionals about sharing illness and treatment information: A qualitative research study. International Journal of Nursing Studies, 83, 91-102
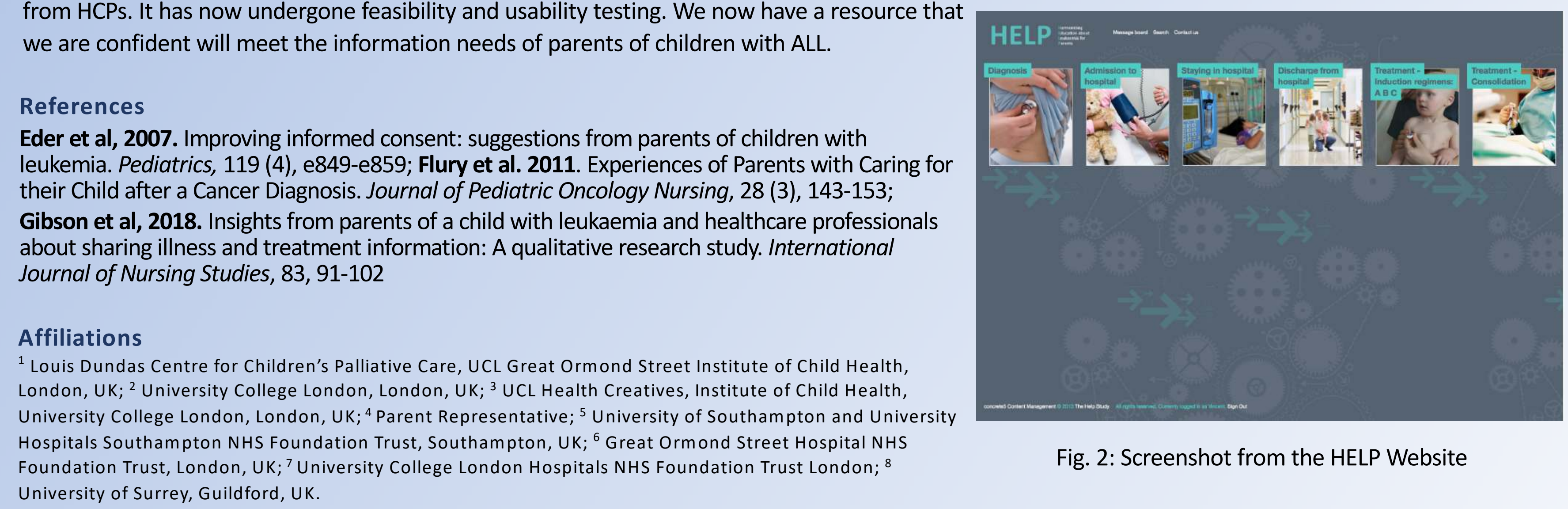